\title{
ESTUDO EPIDEMIOLÓGICO DAS LESÕES TRAUMÁTICAS DE PLEXO BRAQUIAL EM ADULTOS
}

\author{
Leandro Pretto Flores
}

RESUMO - Objetivo: D et e rminar informações epidemiológicas sobre as variáveis relacionadas ao trauma de plexo braquial em adultos. Método: Foram analisados 35 pacientes, de maneira prospectiva, atendidos consecutivamente no período de um ano. Resultados: A maioria das lesões apresentou localização supraclavicular (62\%), sendo 21 lesões por mecanismo de tração (60\%), nove por projétil de arma de fogo $(25 \%)$, três por compressão $(8,5 \%)$ e dois ferimentos cortantes $(5,7 \%)$. Acidentes motociclísticos responderam por $54 \%$ das causas do trauma. A TC-mielografia identificou avulsão radicular em 16 casos (76\%). Melhora neurológica parcial espontânea foi observada em $43 \%$ dos pacientes. Dor neuropática foi observada em 25 casos (71\%) sendo que em 16 (64\%) pôde ser controlada com medicações orais. Conclusão: Os traumas de plexo braquial são mais freqüentemente associados aos mecanismos de tração, sendo comum identificação de avulsão radicular. Em geral produzem dor no membro afetado e estão associados a lesões em outro $\mathrm{s}$ órgãos. Na presente série, a incidência calculada para a população de abrangência foi 1,75/100000/ano.

PALAVRAS-CHAVE: plexo braquial, trauma, epidemiologia.

\section{Epidemiological study of the traumatic brachial plexus injuries in adults}

ABSTRACT - Objective: This study aims to provide information about epidemiological factors related to traumatic brachial plexus injuries in adults. Method: $P$ rospective analysis of 35 consecutive cases, observed in a period of one year. Results: Most of the lesions were supraclavicular $(62 \%)$. Twenty-one cases occurred due to traction $(60 \%), 9$ to gun shot wound $(25 \%), 3$ to compression $(8.5 \%)$ and two perforation/laceration (5.7\%). Motorcycle accidents were the cause of trauma in $54 \%$ of patients. CT myelography demonstrated root avulsion in 16 cases (76\%). Parcial spontaneous neurological recovery was observed in $43 \%$ of the patients. Neuropathic pain occurred in $25(71 \%)$ cases, and the use of some oral intake drugs (as amitriptiline or carbamazepine) controlled it in $64 \%$ of times. Conclusion: Traction is the most frequent mechanism related to brachial plexus injuries, and root avulsions are common in this cases. Pain and concomitant lesions are frequently observed in these group. In this series, the rate of incidence to the local population was 1.75/100000/year.

KEY WORDS: brachial plexus, trauma, epidemiology.

O plexo braquial $(\mathrm{PB})$ é uma região particulamente crítica do sistema nervoso periférico quanto à sua exposição ao trauma. Devido as suas especiais relações anatômicas com as estruturas móveis do pescoço e do ombro, pode ser envolvido quando os vetores de força provocam tração sobre essas estruturas'. Além disso, devido à sua relativa falta de proteção muscular e óssea, pode também ser lesado por ferimentos penetrantes desta região².

Estudos americanos e europeus demonstram que $10 \%$ a $20 \%$ das lesões do sistema nervoso periférico envolvem o plexo braquial. Dessas, de 80 a $90 \%$ são devidas aos traumas auto/motociclísticos, que geram mecanismos de tração sobre o pescoço e ombro². Porém, existem poucos estudos epidemiológicos na literatura mundial a respeito do trauma desta estrutura e, especialmente, existem poucas estatísticas brasileiras sobre o tema.

Assim, nesse estudo avaliam-se os dados epidemiológicos sobre trauma de plexo braquial em adultos em relação à freqüência de diversas variáveis envolvidas, tais como mecanismos, localizações e tipos de lesões, achados aos exames complementares, lesões associadas e dor. Não se pretende, neste momento, aprofundar questões como tratamento, resultados ou prognóstico.

\section{MÉTODO}

Foi realizado estudo prospectivo, com cadastramento e avaliação de todos os pacientes com diagnóstico de lesão

Unidade de Neurocirurgia, Hospital de Base do Distrito Federal, Brasília DF, Brasil.

Recebido 21 Junho 2005, recebido na forma final 17 Agosto 2005. Aceito 6 Outubro 2005.

Dr. Leandro Pretto Flores - SQN 208 / Bloco F / Apt. 604 - 70853-012 Brasília DF - Brasil. E-mail: leandroprettoflores@hotmail.com 
traumática de plexo braquial, procedentes do Distrito Federal (DF), atendidos no Ambulatório de Ciru rgia do Sistema Ne roso Periférico - Unidade de Neurociru rgia do Hospital de Base do DF, no período de maio de 2004 a maio de 2005. Foram incluídos somente os pacientes com idade acima de 12 anos.

Foram obtidas informações sobre os mecanismos do trauma, tempo de ocorrência do acidente, lesões associadas, sintomatologia, apresentação clínica inicial e evolução do quadro clínico, realização de ciru rgias prévias (vasculare s ou ortopédicas), presença de dor neuropática e uso de medicações. Avaliou-se, também, a função motora e sensitiva do membro envolvido, a presença de cicatrizes e de sinais de lesão pré-ganglionar - síndrome de Horner, paralisia de rombóides ou de serrátil anterior - com o objetivo de topografar clinicamente o local da lesão. As lesões supraclaviculares foram classificadas de acordo com a inervação radicular dos músculos envolvidos; e as lesões infraclaviculares, de acordo com alterações semiológicas/eletrofisiológicas características de cordões ou nervos isolados. Os pacientes que queixavam de dor tipo neuropática no membro acometido recebiam prescrição de amitriptilina (50 $\mathrm{mg} / \mathrm{dia}$ ) ou carbamazepina (400 mg/dia).

Estudos el etrofisiológicos foram realizados em todos os casos, no mínimo um mês após o acidente. Os pacientes nos quais a história clínica sugeria lesão provocada por mecanismo de tração/estiramento, eram submetidos à tomografia computadorizada da coluna cervical com injeção de contraste intratecal (TC-mielo). O exame era realizado com secções axiais de $2 \mathrm{~mm}$, iniciando-se em C1 e finalizando na borda inferior de T1. O critério utilizado para a definição de avulsão foi incapacidade de identificação da raiz no nível medular analisado. A presença de pseudomeningocele per se não foi critério para conclusão de arrancamento radicular. Em caso de lesões penetrantes, requisitava-se avaliação de um ciru rgião vascular, com o objetivo de identificação de lesões arteriais ou venosas associadas. Os pacientes com história clínica de lesão por tração ou compressão também realizavam exames radiográficos do tórax, coluna cervical e ombro, para identificação de paralisia de hemicúpula diafragmática, fraturas de elementos vertebrais, fratura de clavícula e/ou escápula.

Os pacientes eram sempre reavaliados em consultas subsequentes. Nestas, avaliava-se a evolução neurológica,

Tabela 1. Correlação entre a localização das lesões e os mecanismos de trauma envolvidos.

\begin{tabular}{lccccc}
\hline & \multicolumn{2}{c}{ Supraclavicular } & \multicolumn{3}{c}{ Infraclavicular } \\
& $\mathrm{n}$ & $\%$ & $\mathrm{n}$ & $\%$ & $\mathrm{p}$ \\
\hline Total & 23 & 62 & 14 & 37 & $<0,05$ \\
Tração & 19 & 51 & 4 & 10,8 & $<0,05$ \\
PAF & 2 & 5,4 & 7 & 18,9 & $<0,05$ \\
Compressão & 1 & 2,7 & 2 & 5,4 & $\mathrm{~ns}$ \\
Laceração & 1 & 2,7 & 1 & 2,7 & $\mathrm{~ns}$ \\
\hline
\end{tabular}

Dois pacientes apresentaram lesões por tração em elementos tanto supra quanto infraclaviculares. o resultados dos estudos eletrofisiológicos e de outros exames radiológicos que eventualmente eram requisitados. $A$ decisão por indicação de cirurgia ou por acompanhamento clínico baseava-se no tipo de mecanismo de lesão, na evolução da recuperação neurológica, nos achados da eletroneuromiografia e nas alterações observadas na TC-mielo.

A análise estatística foi realizada pelo teste do qui-quadrado com correção de Yates, sendo considerados significativos valores com $p<0,05$. Para a realização deste estudo, todas as normas da Declaração de Helsinki para pesquisa em seres humanos foram rigidamente obedecidas e todos participantes assinaram termo de consentimento informado.

\section{RESULTADOS}

No período de um ano, foram atendidos 53 pacientes com lesões traumáticas de nervos periféricos, sendo que 35 (66\%) deles foram de lesões no plexo braquial, o que constitui a presente casuística $(p<$ $0,05)$. Foram $30(85 \%)$ do sexo masculino e $5(15 \%)$ do feminino $(p<0,05)$. A freqüência maior de lesão foi em pacientes com idade inferior a 30 anos $(n=30$; $85 \%, p<0,05)$, já que a distribuição por faixa etária foi de 14 (40\%) pacientes com menos de 20 anos, 16 $(45 \%)$ entre 21 e 30 anos, cinco (14\%) entre 31 e 40 anos e um (2,8\%) com mais de 40 anos.

O tempo decorrido entre a ocorrência do trauma e a primeira avaliação no ambulatório foi de menos de um mês em oito ( $23 \%$ ) pacientes, de um a dois meses em $10(28,5 \%)$, de dois a cinco meses em 10 $(28,5 \%)$ e de mais de cinco meses em sete $(20 \%)$.

Quanto aos mecanismos de lesão, foram estatisticamente mais freqüentes os $21(60 \%)$ casos devido à tração $(p<0,05)$, em relação aos nove $(25 \%)$ por acidente com projétil de arma de fogo (PAF), aos três por mecanismos de compressão $(8,5 \%)$ e aos dois por

Tabela 2. Distribuição das lesões de acordo com as estruturas envolvidas, segundo alterações observadas ao exame físico neurológico e nos estudos eletrofisiológicos.

\begin{tabular}{lcc}
\hline & $\mathrm{n}$ & $\%$ \\
\hline A) Supraclavicular & & \\
C5-C6 & 4 & 19 \\
C5-C6-C7 & 1 & 4,7 \\
C7-C8-T1 & 3 & 14,2 \\
C8-T1 & 2 & 9,5 \\
C5-T1 & 11 & 52,3 \\
B) Infraclavicular & & \\
Cordão lateral & 4 & 28,5 \\
Cordão medial & 2 & 14,2 \\
Cordão posterior & 0 & 0 \\
$\quad$ Múltiplos cordões & 6 & 42,8 \\
$\quad$ Ner vos isolados & 2 & 14,2 \\
\hline
\end{tabular}




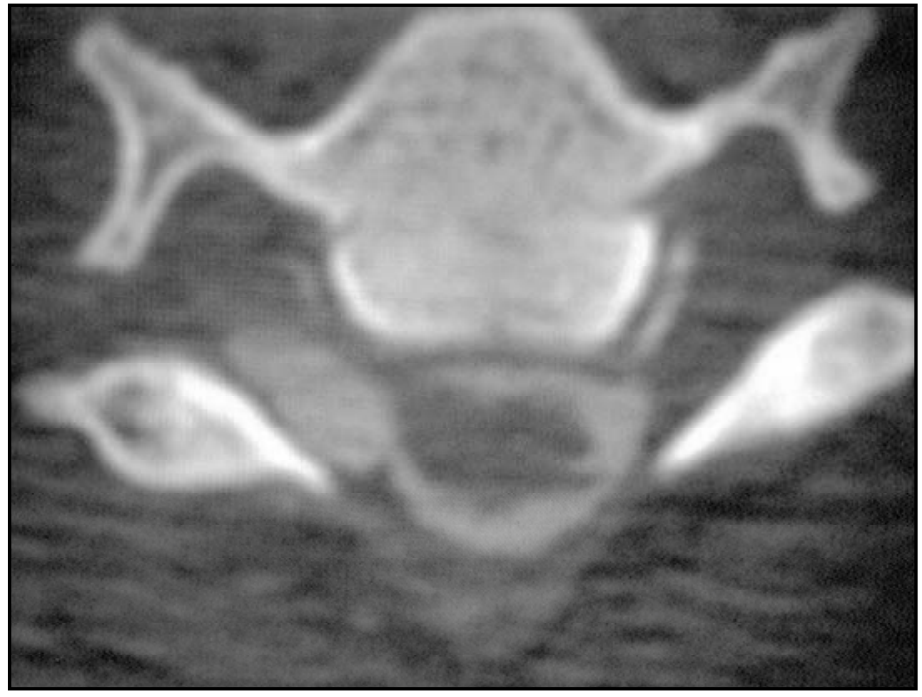

Fig 1. Mielotomografia (TC-mielo) cervical em corte axial: pseudomeningocele direita, onde se identificam raízes nenosas em seu interior. Não é compatível com avulsão radicular.

Fig 2. Mielotomografia (TC-mielo) cervical em corte axial: pseudomeningocele esquerda com ausência de elemen tos nervosos. Compatível com avulsão radicular do nível.

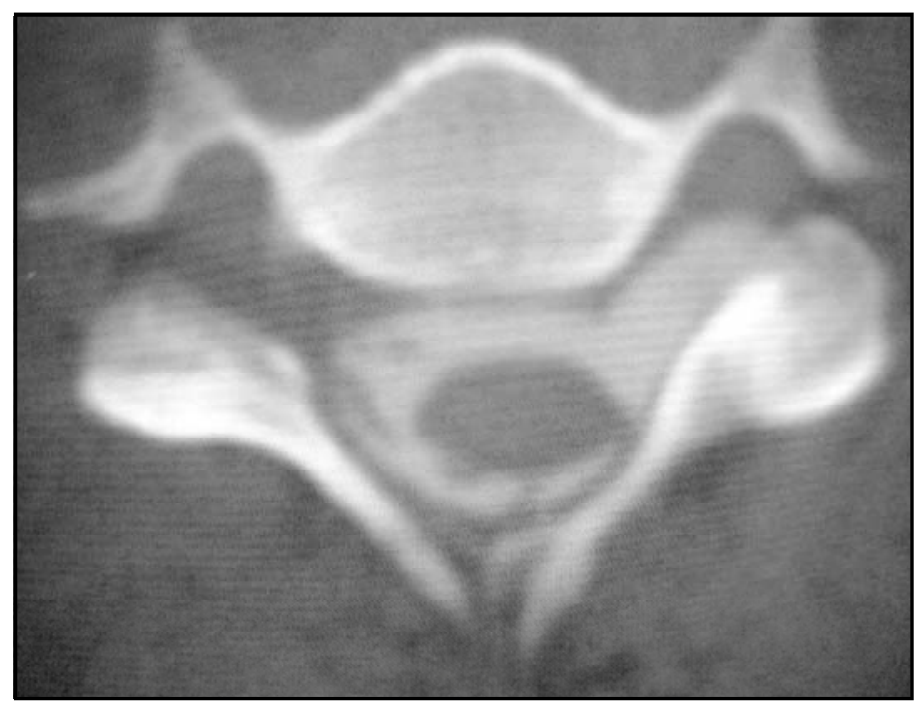

Tabela 3. Tipos de apresentações clínicas observadas.

\begin{tabular}{lccccc}
\hline & Apresentação inicial & \multicolumn{3}{c}{ Evolução* } \\
& $\mathrm{n}$ & $\%$ & $\mathrm{n}$ & $\%$ & $\mathrm{p}$ \\
\hline Paralisia total & 19 & 54 & 11 & 31,4 & $<0,05$ \\
Cordão & 11 & 31 & 14 & 40 & $\mathrm{~ns}$ \\
Erb-Duchene & 3 & 8,5 & 4 & 11,4 & $\mathrm{~ns}$ \\
Dejerine-Klumpke & 1 & 2,8 & 2 & 5,7 & $\mathrm{~ns}$ \\
Outras & 1 & 2,8 & 4 & 11,4 & $\mathrm{~ns}$ \\
Total & 35 & 100 & 35 & 100 & - \\
\hline
\end{tabular}

* Mínimo de quatro meses após o trauma. Obs.: Um paciente apresentou recuperação completa.

ferimentos perfuro-cortante $(5,7 \%)$. Daqueles submetidos a trauma por tração, 18 (85\%) deles sofreram acidentes motociclísticos e os restantes três tiveram acidentes automobilísticos $(p<0,05)$.
Foram encontradas 20 lesões (57\%) no plexo braquial direito e $15(42 \%)$ no esquerdo, mas a diferença não foi estatisticamente significante $(p=0,338)$. Identificaram-se 23 pacientes (62\%) com lesões de localização supraclavicular e 14 infraclavicular (37\%), sendo a diferença significante $(p<0,05)$. Em dois pacientes o mecanismo de tração ocasionou lesões supra e infraclaviculares A Tabela 1 correlaciona a localização das lesões com os mecanismos de trauma. Observase que enquanto a tração esteve mais relacionadas com as lesões supraclaviculares, as lesões por arma de fogo ocasionaram mais lesões infraclaviculares. A Tabela 2 detalha a localização das lesões, utilizando critérios clínicos e eletrofisiológicos para identificação da(s) estrutura(s) lesada(s).

A realização da TC-mielo permitiu a identificação das avulsões radiculares naquelas situações em que 
Tabela 4. Relação entre mecanismo de lesão e: a) evolução da recuperação neurológica espontânea e b) melhora da dor com o uso de amitriptilina ou carbamazepina.

\begin{tabular}{lcccccccc}
\hline & \multicolumn{2}{c}{ Melhora } & \multicolumn{2}{c}{ Sem melhora } & \multicolumn{4}{c}{ Total } \\
& Neuro* & Dor & Neuro* & Dor & $\mathrm{n}$ & $\mathrm{p}$ & Dor & $\mathrm{p}$ \\
\hline Tração & 7 & 9 & 14 & 6 & 21 & $<0,05$ & 15 & $\mathrm{~ns}$ \\
PAF & 7 & 6 & 2 & 2 & 9 & $<0,05$ & 8 & $<0,05$ \\
Compressão & 1 & 1 & 2 & 1 & 3 & $\mathrm{~ns}$ & 2 & $\mathrm{~ns}$ \\
Cortantes & 0 & - & 2 & - & 2 & $\mathrm{~ns}$ & - & - \\
Total & 15 & 16 & 20 & 9 & 35 & - & 25 & - \\
\hline
\end{tabular}

*Neuro, neurológica espontânea.

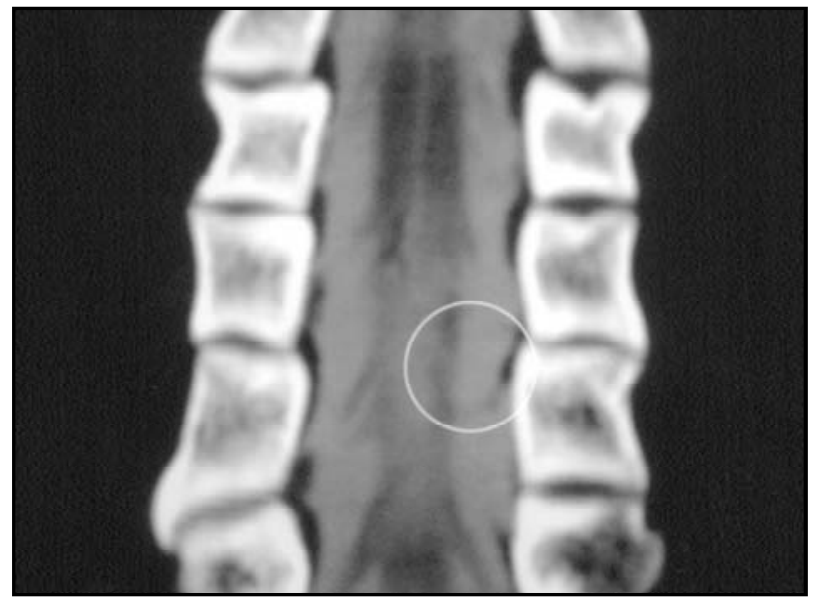

Fig 3. Mielotomografia (TC-mielo) cervical com reconstrução de cortes coronais seriados: observa-se a ausência da raiz de C7 (círculo) sem a ocorrência de pseudomeningocele. Compa tível com diagnóstico de avulsão radicular.

a história do trauma sugeria mecanismo de tração sobre o plexo braquial. Observou-se a avulsão de uma ou mais raízes em 16 (76\%) pacientes dos 21 que realizaram o exame, totalizando 41 raízes lesadas. Dezessete exames demonstravam anormalidades em 51 forames. As principais alterações foram: 35 (73\%) pseudomeningoceles, nas quais em dez (28\%) foi possível identificação de raízes no seu interior (Fig 1) não havia avulsão - e em 25 (71\%) que não demonstravam estruturas nervosas (Fig 2) - critério de avulsão $(p<0,05)$. Em 16 (31\%) forames havia avulsão, apesar de não haver pseudomeningocele (Fig 3). Quanto à localização, observou-se sete avulsões em C5 (17\%), seis (14\%) em C6, oito (19,5\%) em C7, 10 (24\%) em C8 e $10(24 \%)$ em T1.

A eletroneuromiografia foi realizada em todos os pacientes. $O$ exame complementou a avaliação clínica na definição da topografia da(s) lesão(ões) e ainda auxiliou a definir se estas eram pré ou pós-ganglio- na res. Vinte e um casos foram classificados como sendo lesão pós-ganglionar (60\%) e 14 como pré-ganglionar $(40 \%)-p>0,05$. No entanto a CT-mielo identificou avulsão radicular em 16 pacientes. Desta forma os estudos eletrofisiológicos não foram capazes de identificar lesão pré-ganglionar em dois casos (5,7\%).

A Tabela 3 mostra os tipos de apresentações clínicas mais freqüentemente observadas e como estas evoluíram, considerando-se as manifestações clínicas a p resentadas logo após o trauma e, no mínimo, após quatro meses de evolução. Oito pacientes melhoraram da paralisia total, reg redindo para síndromes mais focais, como Erb-Duchene ou Dejerine-Klumpke. Além do déficit motor e sensitivo, 25 pacientes também apresentavam dor no membro superior afetado (71\%). Todos estes foram tratados com medicações orais (amitriptilina $50 \mathrm{mg} / \mathrm{dia}$ ou carbamazepina 400 $\mathrm{mg} / \mathrm{dia}$ ).

A Tabela 4 expõe a evolução do quadro clínico deste grupo de pacientes em relação aos tipos de mecanismos de lesão. Ocorreu melhora neurológica em 15 pacientes ( $43 \%, p=0,253$ - não significativo) e meIhora da dor com uso de antidepressivos tricíclicos ou carbamazepina por período mínimo de um mês de uso, em 16 (64\%, p<0,05). Em relação ao tipo de mecanismo de lesão, observou-se melhora neurológica em $30 \%$ dos casos de tração/estiramento, $77 \%$ dos casos de PAF e $30 \%$ dos casos de compressão. No entanto, esta melhora foi parcial, com exceção de um caso no qual foi considerada completa. Em relação à dor, observou-se melhora em $46 \%$ dos pacientes submetidos à tração, $75 \%$ daqueles por PAF e em todos os casos de compressão. Nenhum paciente com lesão lacerante/perfurante apresentou melhora espontânea neurológica, mas nenhum apresentou quadro doloroso. Os mecanismos de tração, PAF e compressão produziram dor em $71 \%, 88 \%$ e $66 \%$ dos pacientes, respectivamente. 
Tabela 5. Lesões associadas identificadas.

\begin{tabular}{lcc}
\hline & $\mathrm{n}$ & $\%$ \\
\hline Fraturas de ossos longos & 15 & 22 \\
Fraturas de clavícula & 11 & 16 \\
Trauma abdominal cirúrgico & 8 & 12 \\
Pneumotórax & 7 & 10,5 \\
Trauma craniano & 6 & 9 \\
Paralisia de diafragma & 5 & 7,5 \\
Pseudoaneurisma & 4 & 6 \\
Síndrome de Horner & 4 & 6 \\
Lesão vertebral & 3 & 4,5 \\
Fratura de escápula & 3 & 4,5 \\
Total & 66 & 100 \\
\hline
\end{tabular}

Sete pacientes (20\%) já haviam sido submetidos a exploração cirúrgica da região do plexo braquial quando da primeira consulta com a neurocirurgia $(p>0,05)$. Essas haviam sido realizadas para o tratamento de lesões de estruturas vasculares (quatro casos) ou ósseas (três casos). Cinco pacientes haviam sofrido ferimentos por projétil de arma de fogo, um sofreu lesão perfurante infraclavicular e um teve trauma direto na região do ombro - desenvolvendo fraturas de clavícula, processo coracóide e úmero.

Em relação às ciru rgias realizadas para lesões concomitantes em outros órgãos e sistemas, observouse que 19 pacientes $(54,2 \%, p>0,05)$ foram operados, para um total de 23 procedimentos cirúrgicos: oito cirurgias para trauma abdominal, sete drenagens torácicas e oito fixações ortopédicas de ossos longos.

A Tabela 5 apresenta as principais lesões concomitantes identificadas nesta série. Ocorreram 66 lesões em 26 pacientes $(74,2 \%, p<0,05)$.As fraturas de ossos longos e clavícula foram as mais freqüentes. As fraturas de clavícula ocorreram no terço médio em sete pacientes, no terço distal em três e no terço proximal em um. Os quatro casos de pseudoaneurisma observados foram causados por ferimentos por projétil de a rma de fogo em três deles e por lesão de arma branca em um. Das três lesões vertebrais, todas referemse à fratura do processo transverso de $\mathrm{C} 7$ ipsilateral ao plexo braquial lesado.

\section{DISCUSSÃO}

A literatura mundial a respeito de trauma de plexo braquial em geral foca com muita freqüência temas como tratamento e prognóstico, porém infor- mações epidemiológicas são poucas e muito limitadas. Este tipo de informação pode ser muito útil aos serviços de saúde, tanto para o planejamento da assistência ao traumatizado, como para definir manobras de prevenção. Atualmente, não existem taxas de incidência de lesão de plexo braquial na população geral. Mesmo nos Estados Unidos, essa taxa é calculada indiretamente: considerando que a incidência de trauma raquimedular (TRM) é 5 casos/100000 hab/ ano e de trauma crânio-encefálico (TCE) é 200 casos/ 100000 hab/ano3; e considerando que a taxa de ocorrência de lesão de PB é nove vezes menor que TRM e 60 vezes menor do que TCE; a taxa estimada para ocorrência de lesão do PB seria um número entre 0,6 e 3,9 por 100000 habitantes ao ano 4 . Estudando uma amostra de pacientes politraumatizados e excluindo os traumas isolados de membro superior, Midha encontrou uma incidência de 1,2\% deste tipo de lesão em sua série ${ }^{4}$.

O presente estudo baseou-se em uma série de pacientes atendidos consecutivamente no período de um ano, em um ambulatório de referência para lesões do sistema nervoso periférico na rede pública de saúde do DF. Isso permitiu a geração de dados que refletem o que se pode esperar em um serviço de referência em nervos periféricos, quanto ao trauma de plexo braquial. Levando em consideração essa característica, pode-se calcular uma incidência aproximada de lesão do plexo braquial, na região do $D F$, como sendo 1,75 casos/100000 habitantes/ano, já que se identificou 35 casos em um ano, em uma população estimada de dois milhões de pessoas. Esta é apenas uma estimativa, pois não foram incluídos, neste cálculo, os eventuais pacientes atendidos na rede privada ou aqueles atendidos no Hospital Sarah.

Os dados a respeito de gênero e idade confirmam os resultados encontrados na grande maioria dos trabalhos sobre trauma em geral: adultos jovens, do sexo masculino, são os mais atingidos 5 . A diferença identificada no número de ocorrências observadas à $\mathrm{d}$ i reita e à esquerda provavelmente se deve apenas ao acaso. Por outro lado, chama a atenção o elevado númerode casos de lesão do PB em relação ao total de casos de lesão de nervos periféricos - $66 \%$ - que foi estatisticamente significativo. Em geral, a lesão do PB responde por $14 \%$ das lesões traumáticas de nervos ${ }^{6}$. Essa discrepância pode ser explicada por alguns fatores: 1) em geral recebemos pacientes encaminhados de outros serviços; assim, uma deficiência do sistema poderia ser a falta de reconhecimento de lesões de nervos cujos déficits neurológicos são menos evidentes, por médicos pouco familiarizados com o 
exame neurológico - por exemplo, nos casos de lesões nervosas distais ou de lesões de nervos puramente sensitivos; 2) o encaminhamento de casos mais graves para ambulatório especializado, em detrimento dos casos de menor repercussão funcional.

Os mecanismos de tração/estiramento foram os principais responsáveis por lesão do PB nesta série, o que está de acordo com outros autores ${ }^{7}$. Os acidentes motociclísticos responderam pela grande maioria dessas lesões, sendo a causa do trauma em $54 \%$ de todo o grupo de 35 pacientes. Esses foram duas vezes mais freqüentes do que os acidentes por arma de fogo, seis vezes mais do que as compressões e nove vezes mais do que os casos de lesões cortantes. Estudos anteriores confirmam essa tendência: Andrew e Wallance, em 1978, estimaram a freqüência de lesões do PB em acidentes envolvendo motociclistas como sendo 1 para $1000^{8}$; Midha, que havia identificado incidência de 1,2\% de lesão de PB em seu grupo de 4538 politraumatizados, observou uma taxa de $4,2 \%$ para os casos envolvendo acidente motociclístico ${ }^{4}$. Justamente devido ao principal mecanismo ter sido tração, observou-se que as lesões ocorreram com maior freqüência na região supraclavicular, pois as e s t ruturas proximais do plexo em geral são mais susceptíveis a este mecanismo de trauma. Na região infraclavicular, que apresenta maior área de exposição, o principal mecanismo de lesão foram os ferimentos por PAF. As lesões por tração geralmente se associam com pior prognóstico em relação aos outros mecanismos. Isso porque podem gerar avulsão radicular, envolver maior número de estruturas newosas, ou ainda determinar lesões extensas dentro destas estruturas ${ }^{9}$. Portanto, não é surpreendente constatar que as lesões supraclaviculares tenham apre s e $n t a d o$ pior prognóstico em termos de recuperação espontânea do que as infraclaviculares: apenas 30\% dos pacientes cujo trauma ocorreu por tração mostraram algum grau de melhora neurológica clinicamente obsenável - a maioria lesões supraclaviculares, enquanto que $77 \%$ dos pacientes agredidos por PAF mostraram melhora - a maioria lesões infraclaviculares.

A TC-mielo ainda é o exame de eleição para o diagnóstico de avulsão radicular, apresentando valor preditivo positivo de $85 \%$ e negativo de $95 \%{ }^{10}$. O critério para o diagnóstico do arrancamento foi impossibilidade de visualização da raiz. Dessa forma, na presente série, uma observação mais atenta do exame excluiu a presença de avulsão em $28 \%$ das pseudomeningoceles identificadas. Esse exame foi fundamental para a posterior decisão cirúrgica, não apenas para a definição da indicação, mas também para a adequação da tática cirúrgica a ser adotada (necessidade de neurotização intra ou extra-plexal). Epidemiologicamente, observou-se que a maioria dos pacientes vítimas de tração sobre o plexo sofreu avulsão radicular e que, em geral, elas ocorreram em mais de um nível medular. As raízes inferiores - C8 e T1 - foram as mais acometidas, fato este explicado anatomicamente, pois essas raízes não apresentam pontos de ancoragem ao osso do forame intervertebral e desenvolvem um trajeto mais retilíneo em relação às estruturas do ombro, o que facilita a avulsão ${ }^{11}$. A sínd rome de Horner só foi observada em quatro pacientes, apesar da alta freqüência de avulsões de C8/T1; portanto, a não identificação desta alteração semiológica não deve excluir o diagnóstico de avulsão destas raízes.

Os traumas de PB provocam disfunção tanto por lesão neurológica quanto por desenvolvimento de dor no membro afetado. A dor, nesses casos, pode o co rrer por três mecanismos: dor neuropática em lesões pós-ganglionares; dor devido a avulsão radicular; ou dor simpático-mediada ${ }^{12}$. Na presente série, não foi observado nenhum caso de distrofia simpáticoreflexa. Observou-se que a grande maioria dos pacientes - $71 \%$ - apresentou algum quadro doloroso em conseqüência da lesão do plexo. Porém, a maioria mostrou melhora com medidas simples, ou seja, uso de medicações como amitriptilina ou carbamazepi$n a^{13}$. As lesões por tração mostraram pior prognóstico em termos de recuperação da dor, quando comparadas àquelas devido a PAF. Isso provavelmente ocorreu devido à alta incidência de avulsões radiculares identificadas e, nesses casos, pode haver a necessidade da realização de termocoagulação da zona de entrada da raiz dorsal (DREZ) ${ }^{14}$. Mas mesmo entre os casos de tração, observou-se boa resposta aos medicamentos utilizados em $60 \%$ dos pacientes, que foi estatisticamente significante.

A realização de tratamentos prévios para outras complicações do trauma - como fraturas de ossos longos, lesões vasculares ou de vísceras - em geral, além de aumentarem a morbidade desse grupo, atrasou o tratamento da lesão neurológica. Nos pacientes que sofreram exploração da região supra ou infraclavicular para correção de lesões ortopédicas ou vasculares, o atraso foi mais marcante. Muitos desses pacientes apresentaram complicações dessas cirurgias, precisando ocasionalmente mais de um procedimento e assim, aumentando o tempo para o tratamento da lesão neurológica. A alta incidência de ciru rgias para lesões concomitantes - $54,2 \%$ - demonstra que as le- 
sões de PB geralmente estão relacionadas a traumas de maior gravidade. Especificamente com relação aos acidentes por PAF, em muitos casos ocorreram múltiplos ferimentos, observando-se a lesão do PB associada a lesões de tórax, abdome ou de outras extremidades.

Também foi observada alta incidência de lesões concomitantes, especialmente as fraturas de clavículas e ossos logos. Por outro lado, chama a atenção nesta série a baixa incidência de traumatismos cranianos associados $-17 \%$. Midha observou associação com este tipo de complicação em $70 \%$ dos seus pacientes; porém, no estudo desse autor, as lesões de plexo foram identificadas mesmo nos casos muito graves, em pacientes com baixas pontuações na Escala de Coma de Glasgow (em alguns casos as lesões eram definidas com o uso de potencial evocado somato-sensitivo) ${ }^{4}$. Na presente série, foram avaliados somente pacientes atendidos em regime ambulatorial. Pode-se inferir que muitos casos de associação entre traumas cranianos graves e lesões de plexo não foram identificados, em decorrência de seqüelas neurológicas mais graves ou por terem evoluído para óbito. A incapacidade de identificação destes pacientes pode também interferir no cálculo da taxa de incidência real de lesão de PB na população, sendo este mais um viés a ser considerado sobre o resultado que foi previamente apresentado.

Em conclusão, este estudo permitiu observar que os traumas de plexo braquial em adultos, na maioria das vezes, são decorrentes de mecanismos de tração sobreas estruturas nervosas, sendo os acidentes motociclísticos o tipo de atividade mais freqüentemente associada a essas lesões. Esse mecanismo provoca avulsão radicular na maioria dos casos. A maioria dos pacientes apresenta algum tipo de lesão em outros ó rgãos ou sistemas. A melhora neurológica espontânea pôde ser observada em $40 \%$ dos pacientes, estando diretamente relacionada ao tipo de mecanismo de trauma envolvido. Dor de natureza neuropática foi observada em dois terços dos casos, mas a maioria obteve melhora com medicações orais, como antidepressivos tricíclicos ou carbamazepina.

Agradecimentos - $\mathrm{O}$ autor gostaria de agradecer ao Prof. Luiz Augusto Casulari Roxo da Motta pela prestimosa colaboração na revisão dos originais do artigo.

\section{REFERÊNCIAS}

1. Sedel L. Management of supraclavicular lesions: clinical examination, su rgical procedures and results. In Terzis J (ed). Microreconstruction of nerve injuries. Philadelphia: WB Saunders, 1987:385-392.

2. Mumenthaler M. Some clinical aspects of peripheral nerve lesions. Eur Neurol 1969;2:257-268.

3. Kalsbeek WD, McLaurin RL, Harris BSW, Miller JD. The National Head and Spinal Cord Injurie Survey: major findings. J Neurosurg 1980; 53 (Suppl):S19-S31.

4. Midha R. Epidemiology of brachial plexus injuries in a multitrauma poplation. Neurosurgery 1997;40:1182-1189.

5. Cales RH, Trunkey DD. Preventable trauma deaths: a review of trauma care systems development. JAMA 1985;254:1059-1063.

6. Wynn-Parry CR. Brachial plexus injuries. Br J Hosp Med 1984;32: 130-135.

7. Fletcher I. Traction lesions of the brachial plexus. Hand 1969;1:129-136.

8. And rew T, Wallance WA. Do brachial plexus injuries occur at initial impact in motorcyclists? Br Med J 1978;1:1668.

9. Narakas AO. The surgical treatment of traumatic brachial plexus lesions. Int Surg 1980;65:521-527.

10. Carvalho GA, Nikkhah G, Matthies C, Samii M. Diagnosis of root avulsion in traumatic brachial plexus injuries: the value of myelo computed tomography and magnetic resonance imaging. J Neurosurg 1997;86: 69-76.

11. Suterland S. Nerve and nerve injuries. $2^{\text {nd }}$ Edition. Edinburgh: Churchill Livingstone, 1978.

12. Kline DG. Pain of nerve origin. In Kline DG, Hudson AR (eds). Nerve injuries: operative results for major nerve injuries, entrapment and tumors. Philadelphia: WB Saunders, 1995: 513-524.

13. Killian JM, Fromm GH. Carbamazepine in the treatment of neuralgia. Arch Neurol 1968;19:129-136.

14. Samii M, Moringlane JR. Thermocoagulation of dorsal root entry zone for the treatment of intractable pain. Neurosurgery 1984;15:953-956. 